\title{
Clinical Outcome After Replacement of Distal Femur/ Proximal Tibia in a Heterogeneous Patient Cohort: Function Following Tumour, Trauma, and Loosening
}

\author{
TILMAN GRAULICH ${ }^{1}$, CAROLINE KRANZ ${ }^{1}$, CHRISTOPH KORALLUS ${ }^{2}$, \\ MARCUS OERGEL ${ }^{1}$, OMAR-TAREK PACHA ${ }^{1}$, MOHAMED OMAR ${ }^{1}$, \\ EMMANOUIL LIODAKIS ${ }^{1}$, CHRISTIAN KRETTEK $^{1}$ and MARTIN PANZICA ${ }^{3}$ \\ ${ }^{1}$ Trauma Department, Hannover Medical School, Hannover, Germany; \\ ${ }^{2}$ Department of Sports Medicine, Hannover Medical School, Hannover, Germany; \\ ${ }^{3}$ Trauma Department, Klinikum Neustadt am Rübenberge, Neustadt am Rübenberge, Germany
}

\begin{abstract}
Background: Distal femur and proximal tibia replacements as limb-salvage procedures with good outcome parameters for patients with tumours have been broadly described. However, the overall midterm outcome in a mixed, heterogeneous patient collective is still unclear. Patients and Methods: We retrospectively analysed 59 consecutive patients (33 for primary and 26 for revision surgery) between 1998 and 2017. Indication for implantation was tumour $(n=16)$, periprosthetic fracture $(n=14)$, traumatic fracture $(n=14)$, infection $(n=10)$, aseptic loosening $(n=3)$, and pathological fracture $(n=2)$. The mean follow-up duration was 3 years. Clinical functions were evaluated by Toronto Extremity Salvage Score and Knee Society Score. Knee extension and flexion force were measured. Results: The overall survival rate of arthroplasties was $59 \%(n=35)$. Major complications were observed in 36 (61\%) patients. During the follow-up period, 14 (24\%) patients died. We recorded periprosthetic joint infection in 21 (36\%) patients, recurrence of tumour in two (3\%), and aseptic implant failure in three (5\%). The mean Toronto Extremity Salvage Score was 66 33 , and the

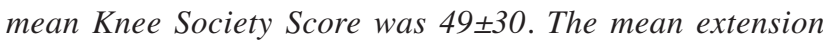
force on the operated side was significantly reduced at $60^{\circ}$ and $180^{\circ}$ compared to the healthy side $(p=0.0151$ and $p=0.0411$, respectively). Conclusion: Distal femur and
\end{abstract}

This article is freely accessible online.

Correspondence to: Tilman Graulich, MD, Trauma Department, Hannover Medical School, Carl-Neuberg-Straße 1, 30625 Hannover, Germany. Tel: +49 17615326723, Fax: +49 5115325877, e-mail: graulich.tilman@mh-hannover.de

Key Words: Knee arthroplasty, revision surgery, tumour, trauma, periprosthetic joint infection, function. proximal tibia replacements showed limited clinical function in a heterogeneous patient collective. Indication for implantation should be considered carefully.

The overall numbers of mega-arthroplasties are increasing. Although clinical function and patient reported outcomes after primary total knee arthroplasty are well described, existing data on revision arthroplasty, such as replacement of the distal femur and proximal tibia, are rare. Indications for revision surgery/patient-specific mega-arthroplasty at the knee are: (i) Primary implantation such as arthroplasty in cases of large bony defects after tumour resection or primary complex fracture; and (ii) revision surgery after failed total knee arthroplasty in cases of aseptic and septic loosening, periprosthetic fracture, pseudarthrosis, or recurrent prosthetic instability (1-4). The incidence of periprosthetic fractures with large bone defects range between $0.3 \%$ and $5.5 \%$ after primary total knee arthroplasty and are seen in up to $30 \%$ cases after revision arthroplasty, with mortality rates as high as $46 \%(1,5)$.

At our clinic, we primarily use the Modular Universal Tumour and Revision System (MUTARS ${ }^{\circledR}$ ) (Implantcast $\mathrm{GmbH}$, Buxtehude, Germany) for large bony defects at the distal femur and proximal tibia, which is a well-established system (6). The stem is made of titanium alloy (TiAl6V4) in the cementless implant and of CoCrMo alloy in the cemented version (1). Although good leg-length alignment and good clinical function can be achieved, implantation is associated with high reoperation rates of $25-50 \%(1,3,5-7)$. The opportunity to return to daily living after distal femur/proximal tibial replacement is impaired by the risk of associated major complications and revision rates as high as $59 \%(5,6,8)$. The most observed complication is a high infection rate $(\sim 34 \%)$ when compared with primary prosthetic joint replacements (5). This is likely due to longer surgery time, larger soft-tissue 
damage, complex biomechanical reconstruction, and larger prosthetic surface area. The total post-operative complication rates range from $25 \%$ to $92 \%$. Functional outcome after megaarthroplasty such as replacement of the distal femur, proximal tibia, or total femur (TFR) is limited (5).

Although published data exist for patients after tumour resection and implantation of a distal femur or proximal tibia replacement, data on a heterogeneous patients collective are still missing $(1,2,9)$. After tumour resection at the knee and implantation of the replacement, a Musculoskeletal Tumor Society score (MSTS) of $66 \%$ and a Toronto Extremity Salvage score (TESS) of 80 points can be expected $(7,9-13)$. These few existing studies have primarily focused on young oncological cohorts (9). However, indications for implantation of distal femur or proximal tibia replacement in cases of revision surgery are on the rise. Therefore, in our study, we calculated the overall survival rate, named complications, and revision strategies to evaluate the post-operative clinical function in patients after megaarthroplasty at the knee. We used validated scores such as TESS and Knee Society Score (KSS) (14). Regarding replacement of distal femur and proximal tibia, because the extensor apparatus and gastrocnemii muscles have to be dissected, we expected a loss of knee extension and flexion force. Thus far, to our knowledge, no data are available on the post-operative force for knee extension. Therefore, we aimed to determine the force reduction.

\section{Patients and Methods}

We retrospectively analysed all patients in our Trauma Department between 1998 and 2017 who underwent a MUTARS replacement of the distal femur or proximal tibia (Implantcast $\mathrm{GmbH}$, Buxtehude, Germany). This system allows modular semi-custom-made distal femur or proximal tibia reconstruction, with adjustable femoral shaft modules and a rotating hinged total knee replacement. Intramedullary stem fixation can be achieved either with or without cement augmentation.

The indications for distal femur-and proximal tibia replacement were tumour in 16, periprosthetic fracture in 14 , infection in 10 , traumatic fracture in 14 , aseptic loosening in three, and pathological fracture in two. The indications for primary implantation were present in $33(56 \%)$ patients and those for implantation in cases of revision surgery were present in 26 (44\%) patients. Major complications were defined as death, revision surgery including amputation, and tumour recurrence. Pre-operative workup included blood cell count and Creactive protein level; in the case of periprosthetic joint infection (PJI), joint aspiration was performed for microbiological analysis and cell count. In the case of positive microbiological results, a two-step revision procedure was performed. At the first stage, a cement spacer was implanted and aggressive debridement was performed; at the second stage, after a 6- to 12-week interval on systemic antibiotics, the reimplantation was performed. Post-operative antibiotics were given until at least the end of the 12-week interval.

Informed consent was obtained from all included participants. The study was approved by the local Ethics Committee (Nr.7957_ BO_S_2018).
Demographics. We included 59 patients [19 (32\%) male and 40 $(68 \%)$ female], with a mean \pm SD age of $65 \pm 20$ years. Implantation of distal femur replacement was performed in 45 patients, proximal tibia replacement was performed in six patients, and both distal femur and proximal tibia replacement was performed in eight patients. The mean body mass index was $27 \pm 6 \mathrm{~kg} / \mathrm{m}^{2}$. Diabetes in 13 and coronary heart disease in 17 were the main comorbidities. Furthermore, only 16 patients were enrolled for the questionnaire survey, and only nine patients were available for knee force measurements.

Scores and function. During outpatient visits, questionnaires were given to the patients and we determined the TESS, MSTS, KSS, and Western Ontario MacMaster questionnaire (WOMAC) scores (14). For TESS (range=0-120), high values indicate a good outcome for evaluation of self-assessed clinical function; for MSTS (range=030 ), high values indicate a good outcome, calculated on the basis of a physical examination by the physician; for KSS (range=0-100), high values indicate a good outcome; and for WOMAC (range=0100), high values indicate a poor outcome.

We performed isokinetic measurements of peak torque for knee extension and flexion using Biodex II Dynamometer (Biodex Medical Systems, Inc., Shirley, NY, USA). The measurement was conducted with three angle speeds at $60 \%$ s (five repetitions), $180 \%$ (five repetitions), and $240 \%$ s (25 repetitions) on both legs in the open kinetic chain. The knee range of motion was limited between 0 and $90^{\circ}$. Patients were placed in a comfortable and upright seated position and were secured using tight pelvic straps to minimise extraneous body movements. After each set of measurements, patients were allowed to rest for $1 \mathrm{~min}$.

Statistical analysis. As the trial was of an exploratory nature, no sample size calculation was performed. Data were summarised using Microsoft Excel ${ }^{\circledR}$ (Microsoft Corporation, Redmond, WA, USA) software, and statistical analysis was performed with IBM SPSS Statistics ${ }^{\circledR}$ Version 25 (IBM Deutschland GmbH, Ehningen, Germany). Data were tested for normal distribution with the Kolmogorov-Smirnov test. For comparative statistics, the parametric t-test and Mann-Whitney $U$-test were used for normally and non-normally distributed variables, respectively. Data are expressed as the mean \pm standard deviation. The standard significance criterion of $\alpha=0.05$ was employed.

\section{Results}

We included 59 patients [19 (32\%) male and 40 (68\%) female], with a mean age of $65 \pm 20$ years at the time of surgery. The mean BMI was $27 \pm 6 \mathrm{~kg} / \mathrm{m}^{2}$, and the mean follow-up was $36 \pm 57$ (range $=1-218$ ) months. Distal femur replacement was performed in $45(76 \%)$ patients, proximal tibia replacement in six (10\%), and both distal and proximal tibia were replaced in eight (14\%). In all, 28 (47\%) patients were operated on the left side and 31 (53\%) on the right side. The indications for distal femur and proximal tibia replacement were tumour in 16 , periprosthetic fracture in 14 , infection in 10, traumatic fracture in 14 , aseptic loosening in three, and pathological fracture in two (Figure 1). The indications for primary implantation were present in 33 

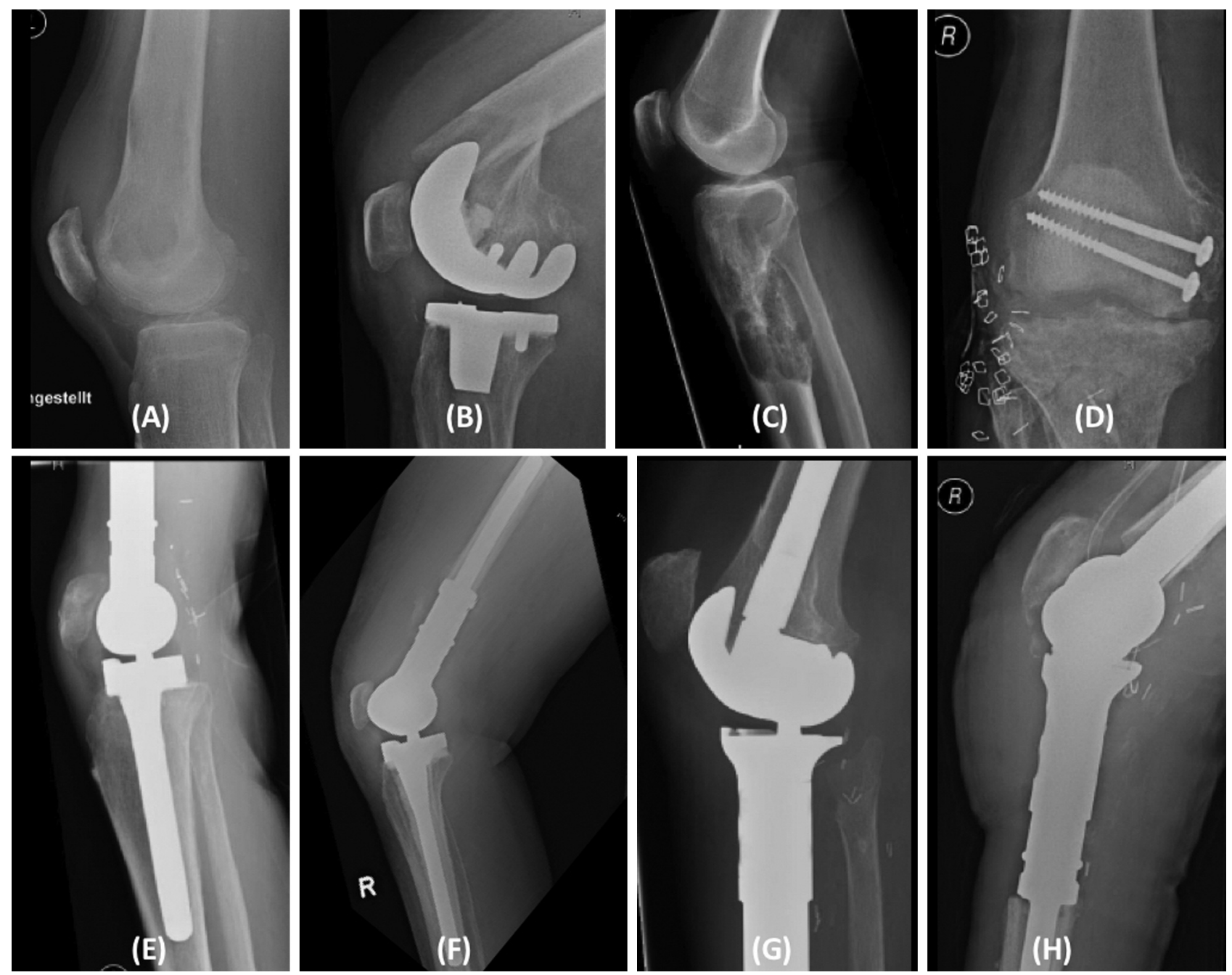

Figure 1. Radiological images for distal femur and proximal tibia implantation. A: A 72-year-old man after treatment for osteomyelitis by distal femur resection and intermediate spacer interposition. B: An 88-year-old woman with periprosthetic fracture treated by distal femur replacement. C: A 70-year-old man with chondrosarcoma of the proximal tibia after resection and proximal tibia replacement. D: A 61-year-old man after fracture fixation, infection, debridement, and implantation of proximal tibia replacement.

(56\%) patients and those for implantation due to revision surgery were present in $26(44 \%)$ patients. All operations were performed by a trained, senior orthopaedic surgeon. The mean operative time was $219 \pm 89 \mathrm{~min}$. In the case of primary surgery, the operative time was $204 \pm 90 \mathrm{~min}$ and in revision surgery, it was $230 \pm 86 \min (p>0.05)$.

Survival rate. The overall rate of arthroplasty survival was 59\% (35 patients). During the follow-up period, 14 (24\%) patients died (51 \pm 54 months). No major complications were observed in 23 (39\%) patients, and major complications were observed in $36(61 \%)$ patients. Among those with major complications, $12(21 \%)$ were successfully treated by revision surgery. Major complications were PJI in 21 (36\%) patients, recurrence of tumour in two (3\%), and aseptic implant failure in three $(5 \%)$. Out of the 21 PJI cases, only six $(30 \%)$ infections were classified as post-operative persistence/recurrence of infection and the remaining 15 $(71 \%)$ were classified as new post-operative infections. Furthermore, 10 out of 21 patients with PJI needed to undergo secondary amputation owing to persistent infection, and seven patients were successfully treated by revision surgery with extensive debridement, explantation, spacer implantation, and reimplantation. Of the remaining four patients, three were treated for chronic infection with antibiotic therapy and died within the follow-up period due to unknown reasons, and one patient died due to septic organ failure. Of the further three patients with implant failure, all 
in vivo $35: 2275-2281(2021)$

Table I. Functional outcome scores with percentage of total score in parentheses.

\begin{tabular}{lcccc}
\hline & Post-operative $(\mathrm{n}=16)$ & Primary surgery $(\mathrm{n}=10$ & Revision surgery $(\mathrm{n}=6)$ & $p$-Value \\
\hline TESS & $66.56 \pm 32.66(55.47 \pm 27.22 \%)$ & $80.10 \pm 28.28(66.75 \pm 23.56 \%)$ & $44.00 \pm 26.42(36.67 \pm 22.02 \%)$ & $\mathbf{0 . 0 3}$ \\
MSTS & $13.88 \pm 7.51(46.25 \pm 25.02 \%)$ & $16.10 \pm 7.16(63.67 \pm 23.87 \%)$ & $10.17 \pm 6.54(33.89 \pm 21.81 \%)$ & 0.14 \\
KSS & $49.06 \pm 29.70$ & $64.50 \pm 24.44$ & $23.33 \pm 17.24$ & $\mathbf{0 . 0 0 4 1}$ \\
WOMAC & $36.31 \pm 26.40(37.83 \pm 27.51 \%)$ & $28.90 \pm 22.21(30.10 \pm 23.13 \%)$ & $48.67 \pm 28.16(50.69 \pm 29.34 \%)$ & 0.17 \\
\hline
\end{tabular}

KSS: Knee Society score; MSTS: Musculoskeletal Tumor Society score; TESS: Toronto Extremity Salvage Score; WOMAC: Western Ontario MacMaster questionnaire. Data are the mean \pm SD. Statistically significant $p$-values are shown in bold.

were successfully treated by revision surgery (two changes of mobile components and one fracture fixation of extensor apparatus).

Clinical outcome. We were only able to include 16 patients for functional scores because of the retrospective nature of the study. The mean values were $66 \pm 33$ for TESS, $14 \pm 7$ for MSTS, $49 \pm 30$ for KSS, and 36 \pm 26 for WOMAC. In primary compared to revision surgery, patients showed significantly better functional scores with TESS of $80 \pm 28$ versus $44 \pm 26$ $(p=0.03)$ and KSS of $64 \pm 24$ versus $23 \pm 17 \quad(p=0.004)$, respectively (Table I). However, in primary operation, the mean flexion was $84^{\circ} \pm 27^{\circ}$, and was not significantly different compared to that after revision surgery $\left(81^{\circ} \pm 20^{\circ}\right)$ $(p=0.343)$. The overall mean flexion was $83^{\circ} \pm 24^{\circ}$. Owing to the heterogeneous patient cohort, pre-operative force measurement was not possible in subjects with fracture and periprosthetic fractures. Therefore, the extension and flexion force, measured in nine patients who were willing or able to participate, was compared with that on the non-operated side. The measured mean extension force at $60^{\circ}$ was $20.26 \pm 12.51$ $\mathrm{Nm}$ on the operated side and $77.46 \pm 58.1 \mathrm{Nm}$ on the nonoperated side $(p=0.0151)$. The mean extension force at $180^{\circ}$ was $23.28 \pm 11.16 \mathrm{Nm}$ on the operated side and $56.51 \pm 40.81$ $\mathrm{Nm}$ on the non-operated side $(p=0.0411)$. The mean extension force at $240^{\circ}$ was not statistically different. The mean flexion force at $60^{\circ}, 180^{\circ}$, and $240^{\circ}$ showed no significant differences (Table II, Figure 2). No significant differences in the muscle diameter were observed $(p>0.05)$.

\section{Discussion}

In our study, we retrospectively analysed patients who underwent replacement of the distal femur or proximal tibia. Compared to earlier studies, besides patients with tumour, we included patients after periprosthetic fractures, infection, traumatic fracture, aseptic loosening, and pathological fracture (9). As this is an outstanding difference, the data provided are unique and have to be interpreted on this basis. The two major findings of this study were as follows: Firstly,
Table II. Force measurement for knee extension and knee flexion ( $n=9)$.

\begin{tabular}{|c|c|c|c|}
\hline & \multicolumn{2}{|c|}{ Force $(\mathrm{Nm})$} & \multirow[b]{2}{*}{$p$-Value } \\
\hline & Operated side & Non-operated side & \\
\hline Extension at $60 \% \mathrm{~s}$ & $20.26 \pm 12.51$ & $77.46 \pm 58.10$ & 0.0151 \\
\hline Extension at $180 \% \mathrm{~s}$ & $23.28 \pm 11.16$ & $56.51 \pm 40.81$ & 0.0411 \\
\hline Extension at $240 \% \mathrm{~s}$ & $27.78 \pm 17.17$ & $49.34 \pm 36.60$ & 0.1508 \\
\hline Flexion at $60 \% \mathrm{~s}$ & $32.47 \pm 26.31$ & $52.63 \pm 42.20$ & 0.2683 \\
\hline Flexion at $180^{\circ} / \mathrm{s}$ & $24.33 \pm 21.76$ & $43.63 \pm 35.19$ & 0.2056 \\
\hline Flexion at $240^{\circ} / \mathrm{s}$ & $23.51 \pm 20.67$ & $48.94 \pm 39.32$ & 0.1249 \\
\hline
\end{tabular}

Statistically significant $p$-values are shown in bold.

at the 3-year follow-up after implantation, 59\% patients were successfully treated by distal femur/proximal tibia replacement. Secondly, a significant reduction of $59-75 \%$ in the extension force was recorded compared to the nonoperated side, with an overall limited clinical outcome as measured by clinical function scores.

Our complication rate of $61 \%$ is comparable to the rate of $59 \%$ reported in the literature $(5,6,8)$. These data are also comparable to our own study cohort with patients after total femur replacements and complication rates up to $50 \%$ (5). Interestingly, although we did expect an even worse revision rate than in other studies, owing to an older and more complex patient cohort, we did not find this to be the case. However, comparing our data to those described by Tunn et al. (9) in a cohort of patients after tumour resection, with a complication rate of $28 \%$ and an explantation rate of $24 \%$, we observed the aforementioned expected worse function most likely due to the higher degree of patients with a complex patients history. Differences in distal femur replacement and proximal tibia replacement were not observed by Tunn et al.

Based on functional outcome, a reduction in MSTS and TESS scores of approximately $50 \%$ was expected at a mean follow-up of 6.5 years (9). Compared to these data of Tunn et al., our data showed overall worse MSTS score (46\% vs. 


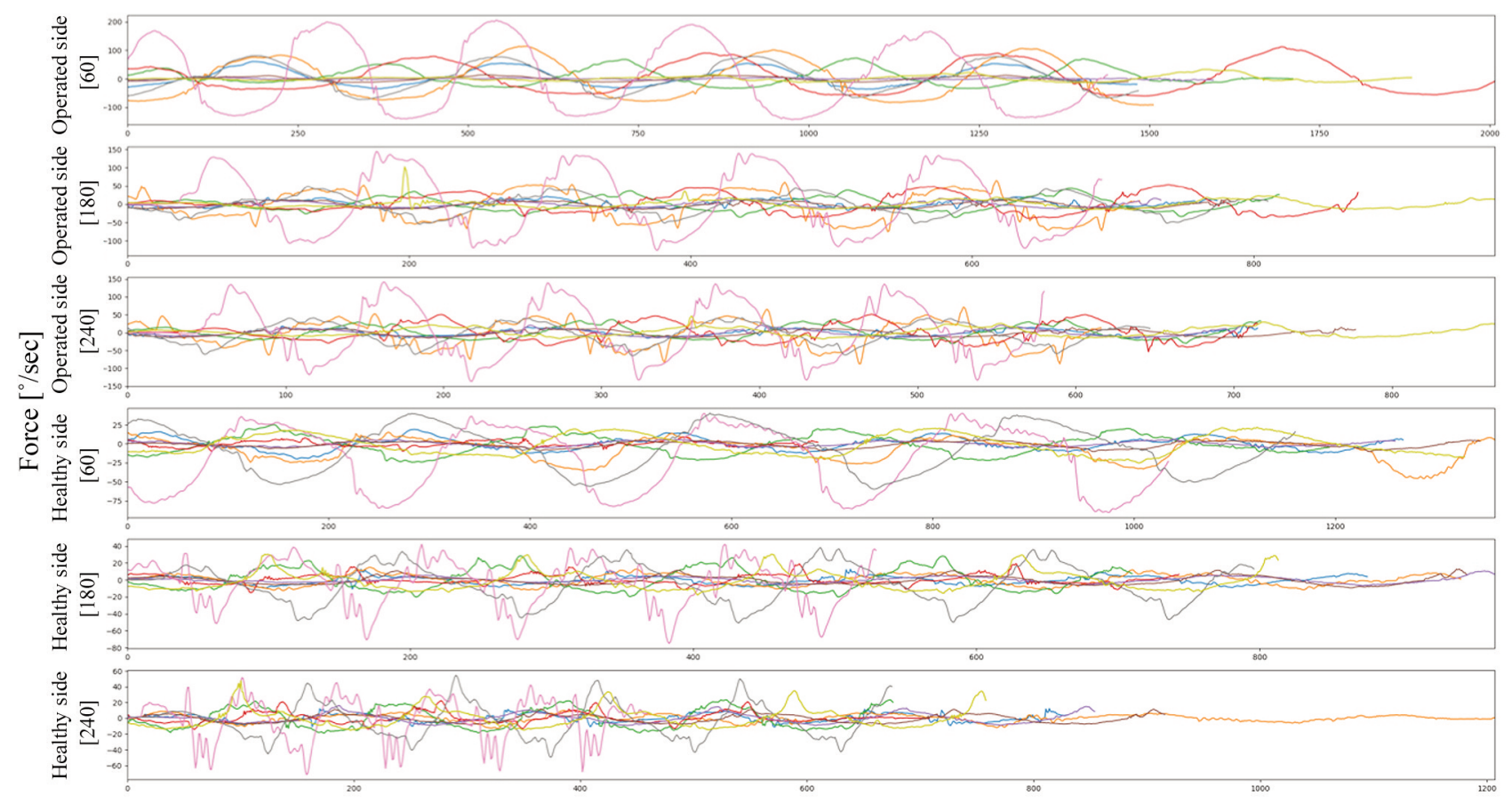

Time [seconds]

Figure 2. Measured extension and flexion force using the Biodex II Dynamometer

$77 \%)$ and TESS (66\% vs. 82\%). However, a subgroup analysis of primary surgery cases showed that the TESS score is very much comparable to the expected data from the literature $(80 \% v s .82 \%)$ and worse in cases of revision surgery (44\% vs. $82 \%)$. Therefore, we believe that the overall worse results in patients' self-reported outcome scores can be explained by the heterogeneous cohort. Our results indicate that although good results can be expected in younger patients undergoing surgery for primary tumour, worse results should be expected in the case of revision surgery in the elderly.

Furthermore, the analysis of the muscle force for extension and flexion showed differences between the nonoperated and operated sides, with a reduction in extension force. Although we would have expected a significant reduction of both extension and flexion forces, we believe that the non-significant reduction in flexion force is a reflection of the small number of patients from whom force measurements were obtained. Nevertheless, a trend towards reduced flexion force was observed.

An alternative therapeutic option in the case of stable periprosthetic fractures (Type I and II, per Rorabeck) would be plate fixation such as that studied by Lotzien et al. (15, 16). They reported a mortality rate of $26.7 \%$ after periprosthetic distal femur fractures in their study of 45 patients with a mean age of 74 years (15). The mortality rate of $23 \%$ found in our study was consistent with this. Mortality was associated with higher values in the Charlson Comorbidity Index, again showing the impact of this injury in old and morbid patients (15). Furthermore, functional outcome was analysed by Lotzien et al. and measured by the American Short Musculoskeletal Function Assessment Questionnaire, with scores ranging from 0 to 100 , with high values indicating poor function (15). A post-operative score of 41.5 points was observed. Although these data are difficult to compare with our study owing to the different scoring system, an overall moderate outcome can be seen in functional score per Lotzien et al.'s study (15). This might lead to the theoretical consequence of favouring primary arthroplasty in the case of periprosthetic fractures, especially in the elderly, with the advantage of direct mobilisation with full weightbearing. However, comparison of both methods by Hoellwarth et al. showed no differences in a retrospective follow-up in patients $>55$ years with regard to mortality and complication rates (17). This seems to be confirmed in further retrospective evaluation studies of both procedures in the literature (18-20). To further evaluate these two therapeutic options, the prospectively, randomised trial - Geriatric Distal Femur Fixation Versus Replacement (DIFFIR) - is ongoing (ClinicalTrials.gov Identifier: NCT04076735). 
Limitations. Although a post-hoc power analysis for extension force at $60^{\circ}$ showed good power of $75 \%$, that for flexion force at $60^{\circ}$ revealed only poor power $(26 \%)$. A posthoc sample size calculation showed the need for 140 patients in total. Therefore, we believe that our data on force measurement provide a hint regarding reduced post-operative power. However, more data should be collected to validate expectations on post-operative function.

\section{Conclusion}

Implantation of a distal femur/proximal tibia replacement is a limb-salvage procedure with an even poorer outcome in a heterogeneous patient cohort than in patients after primary tumour resection. Subjective patient scores showed moderate outcome and a tremendous reduction of extension and flexion power compared to the non-operated side. In the case of primary surgery, better functional results can be expected than in cases of revision surgery.

\section{Conflicts of Interest}

The Authors have no conflicts of interest to declare that are relevant to the contents of this article.

\section{Authors' Contributions}

Conceptualisation: T. Graulich, E. Liodakis, C. Krettek, M. Panzica; Methodology: T. Graulich, C. Kranz, C. Korallus, M. Oergel, T. Omar-Pacha, M. Omar, E. Liodakis, C. Krettek, M. Panzica; Formal analysis and investigation: T. Graulich, E. Liodakis, C. Krettek, M. Panzica; Methodology: T. Graulich, C. Kranz, C. Korallus, M. Oergel, T. Omar-Pacha, M. Omar, E. Liodakis, C. Krettek, M. Panzica; Writing - original draft preparation: T. Graulich; Writing review and editing: T. Graulich, E. Liodakis, C. Krettek, M. Panzica; Methodology: T. Graulich, C. Kranz, C. Korallus, M. Oergel, T. Omar-Pacha, M. Omar, E. Liodakis, C. Krettek, M. Panzica; Funding acquisition: none; Resources: T. Graulich, E. Liodakis, C. Krettek, M. Panzica; Methodology: T. Graulich, C. Kranz, C. Korallus, M. Oergel, T. Omar-Pacha, M. Omar, E. Liodakis, C. Krettek, M. Panzica; Supervision: T. Graulich, M. Omar, E. Liodakis, C. Krettek, M. Panzica.

\section{References}

1 Heisel C, Kinkel S, Bernd L and Ewerbeck V: Megaprostheses for the treatment of malignant bone tumours of the lower limbs. Int Orthop 30(6): 452-457, 2006. PMID: 16967279. DOI: 10.1007/s00264-006-0207-7

2 Kinkel S, Lehner B, Kleinhans JA, Jakubowitz E, Ewerbeck V and Heisel C: Medium to long-term results after reconstruction of bone defects at the knee with tumor endoprostheses. J Surg Oncol 101(2): 166-169, 2010. PMID: 19924724. DOI: $10.1002 /$ jso. 21441

3 Zajonz D, Zieme A, Prietzel T, Moche M, Tiepoldt S, Roth A, Josten C, von Salis-Soglio GF, Heyde CE and Ghanem M: Periprosthetic joint infections in modular endoprostheses of the lower extremities: a retrospective observational study in 101 patients. Patient Saf Surg 10: 6, 2016. PMID: 26865860. DOI: 10.1186/s13037-016-0095-8

4 Raschke MJ, Stange R and Kösters C: Treatment of periprosthetic and peri-implant fractures: modern plate osteosynthesis procedures. Unfallchirurg 115(11): 1009-1021, 2012. PMID: 23143032. DOI: 10.1007/s00113-012-2317-6

5 Graulich T, Steimer D, Zhang D, Omar M, Weber-Spickschen S, Krettek $\mathrm{C}$ and Panzica M: High complication and revision rates after total femoral replacement: a retrospective single center analysis of indication, function, and complication. Arch Orthop Trauma Surg 139(7): 913-920, 2019. PMID: 30687872. DOI: 10.1007/s00402-019-03130-w

6 Gebert C, Wessling M, Götze C, Gosheger G and Hardes J: The Modular Universal Tumour And Revision System (MUTARS ${ }^{\circ}$ ) in endoprosthetic revision surgery. Int Orthop 34(8): 1261-1265, 2010. PMID: 20379815. DOI: 10.1007/s00264-010-1007-7

7 Natarajan MV, Balasubramanian N, Jayasankar V and Sameer M: Endoprosthetic reconstruction using total femoral custom mega prosthesis in malignant bone tumours. Int Orthop 33(5): 13591363, 2009. PMID: 19259671. DOI: 10.1007/s00264-009-0737-x

8 Gerdesmeyer L, Töpfer A, Kircher J, Grundei H and Diehl P: [The modular MML revision system in knee revision and tumor arthroplasty]. Orthopade 35(9): 975-981, 2006. PMID: 16897030. DOI: 10.1007/s00132-006-0982-2

9 Tunn PU, Pomraenke D, Goerling U and Hohenberger P: Functional outcome after endoprosthetic limb-salvage therapy of primary bone tumours - a comparative analysis using the MSTS score, the TESS and the RNL index. Int Orthop 32(5): 619-625, 2008. PMID: 17701173. DOI: 10.1007/s00264-007-0388-8

10 Jones KB, Griffin AM, Chandrasekar CR, Biau D, Babinet A, Deheshi B, Bell RS, Grimer RJ, Wunder JS and Ferguson PC: Patient-oriented functional results of total femoral endoprosthetic reconstruction following oncologic resection. J Surg Oncol 104(6): 561-565, 2011. PMID: 21695701. DOI: 10.1002/ jso. 22003

11 Ruggieri P, Bosco G, Pala E, Errani C and Mercuri M: Local recurrence, survival and function after total femur resection and megaprosthetic reconstruction for bone sarcomas. Clin Orthop Relat Res 468(11): 2860-2866, 2010. PMID: 20680532. DOI: 10.1007/s11999-010-1476-4

12 Enneking WF, Dunham W, Gebhardt MC, Malawar M and Pritchard DJ: A system for the functional evaluation of reconstructive procedures after surgical treatment of tumors of the musculoskeletal system. Clin Orthop Relat Res (286): 241246, 1993. PMID: 8425352.

13 Davis AM, Wright JG, Williams JI, Bombardier C, Griffin A and Bell RS: Development of a measure of physical function for patients with bone and soft tissue sarcoma. Qual Life Res 5(5): 508-516, 1996. PMID: 8973131. DOI: 10.1007/BF00540024

14 Giesinger JM, Hamilton DF, Jost B, Behrend H and Giesinger K: WOMAC, EQ-5D and Knee Society Score thresholds for treatment success after total knee arthroplasty. J Arthroplasty 30(12): 21542158, 2015. PMID: 26160647. DOI: 10.1016/j.arth.2015.06.012

15 Lotzien S, Hoberg C, Hoffmann MF and Schildhauer TA: Clinical outcome and quality of life of patients with periprosthetic distal femur fractures and retained total knee arthroplasty treated with polyaxial locking plates: a single-center experience. Eur J Orthop Surg Traumatol 29(1): 189-196, 2019. PMID: 29931530. DOI: 10.1007/s00590-018-2266-z 
16 Rorabeck $\mathrm{CH}$ and Taylor JW: Classification of periprosthetic fractures complicating total knee arthroplasty. Orthop Clin North Am 30(2): 209-214, 1999. PMID: 10196422. DOI: 10.1016/s0030-5898(05)70075-4

17 Hoellwarth JS, Fourman MS, Crossett L, Goodman M, Siska P, Moloney GB and Tarkin IS: Equivalent mortality and complication rates following periprosthetic distal femur fractures managed with either lateral locked plating or a distal femoral replacement. Injury 49(2): 392-397, 2018. PMID: 29208310. DOI: 10.1016/j.injury.2017.11.040

18 Drew JM, Griffin WL, Odum SM, Van Doren B, Weston BT and Stryker LS: Survivorship after periprosthetic femur fracture: factors affecting outcome. J Arthroplasty 31(6): 1283-1288, 2016. PMID: 26935943. DOI: 10.1016/j.arth.2015.11.038
19 Leino OK, Lempainen L, Virolainen P, Sarimo J, Pölönen T and Mäkelä KT: Operative results of periprosthetic fractures of the distal femur in a single academic unit. Scand J Surg 104(3): 200207, 2015. PMID: 25332221. DOI: 10.1177/1457496914552343

20 Ruder JA, Hart GP, Kneisl JS, Springer BD and Karunakar MA: Predictors of functional recovery following periprosthetic distal femur fractures. J Arthroplasty 32(5): 1571-1575, 2017. PMID: 28131543. DOI: 10.1016/j.arth.2016.12.013

Received April 17, 2021

Revised May 4, 2021

Accepted May 5, 2021 